ADDENDUM

doi:10.1038/nature13077

\section{Addendum: Optical-field-induced current in dielectrics}

Agustin Schiffrin, Tim Paasch-Colberg, Nicholas Karpowicz, Vadym Apalkov, Daniel Gerster, Sascha Mühlbrandt, Michael Korbman, Joachim Reichert, Martin Schultze, Simon Holzner, Johannes V. Barth, Reinhard Kienberger, Ralph Ernstorfer, Vladislav S. Yakovlev, Mark I. Stockman \& Ferenc Krausz

Nature 493, 70-74 (2013); doi:10.1038/nature11567

Calculations in this Letter ${ }^{1}$ and in ref. 2 are sensitive to the dipole matrix elements, $Z_{v i, c j}(i, j=1,2)$, describing the transitions between the two uppermost valence ( $v 1$ and $v 2$ ) and two lowest conduction ( $c 1$ and $c 2$ ) bands (see Supplementary Information to ref. 1). They have resulted in good agreement with all observations of both experiments for $Z_{v 1, c 1}=3 \AA$, $Z_{v 1, v 2}=Z_{c 1, c 2}=2 \AA$ and $Z_{v 2, c 2}=0$. By mistake, Fig. $1 \mathrm{~b}$ and Supplementary Fig. 4 of ref. 1 show adiabatic levels of silica for $Z_{v 1, c 1}=1 \AA\left(Z_{v 1, v 2}\right.$, $Z_{c 1, c 2}$ and $Z_{v 2, c 2}$ are correct and set as above). Here we correct this error in Fig. 1 by plotting the adiabatic levels of silica for the value of $Z_{v 1, c 1}=3 \AA$ that we actually used. This corrected level diagram prompts a correction of the qualitative picture mediated by Fig. $1 \mathrm{~b}$ and Supplementary Figs 4 and 6 of ref. 1, aiming at a qualitative insight of the strong-field optical phenomena reported in refs 1 and 2 . The corrected picture suggests an analogy to a phenomenon known in semiconductor physics for more than two decades. We summarize these findings below. The experimental and quantitative results presented in refs 1 and 2 stand as published, as do the conclusions derived directly from them.

As we have argued in the Supplementary Information to ref. 1 for slowly varying fields of strengths $|F| \approx 1-3 \mathrm{~V}^{-1}$, electronic states are predicted to undergo Wannier-Stark localization ${ }^{3}$ within approximately one unit cell of the wide-gap material—silica-under scrutiny. At a field strength where a level anticrossing denoted by $\Delta l$ occurs in Fig. 1 , Zener tunnelling ${ }^{4}$ between the localized Wannier-Stark states across $|\Delta l|-1$

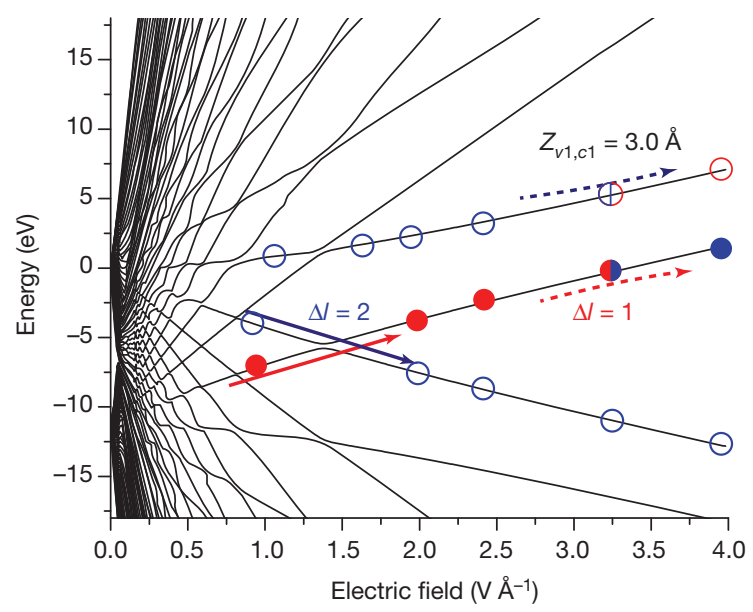

Figure $1 \mid$ Electronic transitions between the valence and conduction bands of silica in a strong optical field. Schematic of the adiabatic energy levels as a function of external electric field, for interband dipole moment $Z_{v 1, c 1}=3 \AA$. A crystal with a thickness of 50 unit cells in the field direction is considered. The anticrossings with $\Delta l=1$ and $\Delta l=2$ are indicated. Red circles denote levels with the valence-band wavefunctions; blue circles denote levels with the conduction-band wavefunctions. Closed circles indicate filled states and open circles indicate empty states. The arrows indicate level anticrossings passed diabatically (solid crossed arrows) and adiabatically (dashed parallel arrows). unit cells is expected to occur, with a rate equal to the anticrossing energy gap (in frequency units) $\Delta E_{l} /(2 \pi \hbar)$. As Fig. 1 reveals, for $|\Delta l| \geq 2$, this rate is very small with respect to the carrier frequency, $\omega_{L} / 2 \pi$, of the optical field (where subscript ' $L$ ' is for 'laser'). Hence, all these anticrossings are passed diabatically in the oscillating strong laser field. The system follows the diabatic terms (shown by the solid crossed arrows in Fig. 1) as the field increases or decreases without significant transfer of population between the valence band and the conduction band. This is because the system passes the anticrossings too quickly for Zener tunnelling to occur. Such an anticrossing is mostly inconsequential (almost 'ignored' by the system).

As the corrected level diagram in Fig. 1 reveals, the gap becomes very large, with $\Delta E_{l} / \hbar>>\omega_{\mathrm{L}}$, for the ultimate level anticrossing at $|\Delta l|=1$ occurring when the field amplitude approaches and exceeds the critical field strength $F_{\text {crit }}=\Delta_{\mathrm{g}} / e a$ where $\Delta_{\mathrm{g}} \approx 9 \mathrm{eV}$ is the bandgap of silica, $e$ is the charge on the electron and $a$ is the lattice constant). The large anticrossing gap implies a large probability of Zener tunnelling between the valence band and conduction band states, which are Wannier-Starklocalized at the nearest neighbours. This anticrossing is passed predominantly adiabatically, as indicated by the dashed parallel arrows in Fig. 1. This leads necessarily to the lower anticrossing level gradually changing its wavefunction from valence band to conduction band (the well known exchange of quantum numbers in adiabatic anticrossings) while remaining fully occupied. This gradual change can be quantified by projecting the actual filled, adiabatic field-modified valence band states to zero-field conduction-band states as given by Supplementary equation (10) in ref. 2 . This projection results in a transient population of virtual conduction-band electrons (the evolution of which is shown in figure $3 \mathrm{~d}$ of ref. 2 ) and the corresponding virtual valence-band holes.

These are virtual carriers similar to those responsible for virtual photoconductivity in semiconductors theoretically predicted ${ }^{5-7}$ and experimentally observed ${ }^{8}$ over two decades ago. However, the analogy is incomplete. In our case, the virtual carriers are produced by a nonresonant (mostly adiabatic), strong-field (and hence, necessarily, nonperturbative) excitation, in contrast with early work $^{5-8}$, where they were excited via a near-resonant perturbative excitation. These virtual carriers make the system more polarizable in a static electric field ${ }^{5-8}$ and likewise at optical frequencies. If so, they can account for both the transient reflectivity shown in figure $3 \mathrm{~b}$ of ref. 2 and the transient current displayed in figure 3 of ref. 1 . In both cases, a strong few-cycle field nonlinearly enhances the strong-field polarization of the dielectric by creating virtual carriers. These respond with increased excursions to a weaker optical field, which probes the resultant enhancement in reflectivity and separates the charges that-after being trapped in the electrodes or within the insulator-subsequently induce a current through an external circuit in refs 2 and 1, respectively. Note that our quantitative quantum model ${ }^{1,2}$ fully takes into account this virtual-carrier mechanism.

We emphasize that our original interpretation ${ }^{1,2}$ of the strong field causing a reversible quantum transition (that we name 'semi-metallization') from an insulating state to one with increased conductivity is valid. It allows one to gain a useful independent insight into highly non-equilibrium non-perturbative processes in strong ultrafast optical fields (see also ref. 9). We note that recent $a b$ initio calculations ${ }^{10}$ for insulators in strong fields are interpreted in terms of the field-induced insulator to metal transition.

In conclusion, we have provided a unified qualitative explanation for the optical-field-induced transient reflectivity ${ }^{2}$ and current ${ }^{1}$ in terms of virtual electron-hole pairs generated by a strong optical field. In sharp contrast with virtual photoconductivity of semiconductors ${ }^{5-8}$, these effects are induced by photons far below the bandgap and respond nearly adiabatically - in a highly nonlinear, nonperturbative fashionto the instantaneous field, rather than its cycle-averaged intensity as in refs 5-8, as it approaches $F_{\text {crit }}$. This opens up the prospect of exploiting the new effects for advancing solid-state high-speed metrology and signal processing towards the petahertz frontier. 
1. Schiffrin, A. et al. Optical-field-induced current in dielectrics. Nature 493, 70-74 (2013).

2. Schultze, M. et al. Controlling dielectrics with the electric field of light. Nature $\mathbf{4 9 3}$, 75-78 (2013)

3. Wannier, G. H. Elements of Solid State Theory (Cambridge Univ. Press, 1959).

4. Zener, C. A theory of the electrical breakdown of solid dielectrics. Proc. R. Soc. Lond. Ser. A 145, 523-529 (1934).

5. Yamanishi, M. Field-induced optical nonlinearity due to virtual transitions in semiconductor quantum well structures. Phys. Rev. Lett. 59, 1014-1017 (1987).

6. Chemla, D. S., Miller, D. A. B. \& Schmitt-Rink, S. Generation of ultrashort electrical pulses through screening by virtual populations in biased quantum wells. Phys. Rev. Lett. 59, 1018-1021 (1987).

7. Yablonovitch, E., Heritage, J. P., Aspnes, D. E. \& Yafet, Y. Virtual photoconductivity. Phys. Rev. Lett. 63, 976-979 (1989).

8. Hu, B. B., Zhang, X. C. \& Auston, D. H. Terahertz radiation induced by subband-gap femtosecond optical excitation of GaAs. Phys. Rev. Lett. 67, 2709-2712 (1991).

9. Krausz, F. \& Stockman, M. I. Attosecond metrology: from capturing electrons toward speeding up signal processing. Nature Photon 8, 205-213 (2014).

10. Wachter, G. et al. Ab-initio simulation of optical-field induced currents in dielectrics. Preprint at http://arxiv.org/abs/1401.4357 (2014).

Correspondence should be addressed to A.S. (agustin.schiffrin@mpq.mpg.de), M.I.S. (mstockman@gsu.edu) or F.K. (ferenc.krausz@mpq.mpg.de). 